\title{
Iterative Fault Tolerant Control Based on
} Stochastic Distribution

Skaf, Zakwan and Al-Bayati, Ahmad and Wang, Hong and Wang, Aiping

2011

MIMS EPrint: 2012.10

Manchester Institute for Mathematical Sciences

School of Mathematics

The University of Manchester

\footnotetext{
Reports available from: http://eprints.maths.manchester.ac.uk/

And by contacting: The MIMS Secretary

School of Mathematics

The University of Manchester

Manchester, M13 9PL, UK
} 


\title{
Iterative Fault Tolerant Control Based on Stochastic Distribution
}

\author{
Zakwan Skaf, Ahmad AI-Bayati, Hong Wang and Aiping Wang
}

\begin{abstract}
A new design of a fault tolerant control (FTC)based an adaptive, fixed-structure PI controller, with constraints on the state vector for nonlinear discrete-time system subject to stochastic non-Gaussian disturbance is studied. The objective of the reliable control algorithm scheme is to design a control signal such that the actual probability density function (PDF) of the system is made as close as possible to a desired PDF, and make the tracking performance converge to zero, not only when all components are functional but also in case of admissible faults. A Linear Matrix Inequality (LMI)-based FTC method is presented to ensure that the fault can be estimated and compensated for. A radial basis function (RBF) neural network is used to approximate the output PDF of the system. Thus, the aim of the output PDF control will be a RBF weight control with an adaptive tuning of the basis function parameters. The key issue here is to divide the control horizon into a number of equal time intervals called batches. Within each interval, there are a fixed number of sample points. The design procedure is divided into two main algorithms, within each batch, and between any two adjacent batches. A P-type ILC law is employed to tune the parameters of the RBF neural network so that the PDF tracking error decreases along with the batches. Sufficient conditions for the proposed fault tolerance are expressed as LMIs. An analysis of the ILC convergence is carried out. Finally, the effectiveness of the proposed method is demonstrated with an illustrated example.
\end{abstract}

\section{INTRODUCTION}

There are many stochastic systems in practice whose outputs are the PDF of the system output[1], rather than the actual output values. For such cases, the measured output PDFs can be used as an output for the feedback control. Such types of stochastic systems are called Stochastic Distribution Control (SDC) systems [1]. Practical examples of SDC systems in industrial applications include: Combustion flame distribution processes [2], and the wet-end of paper-making [1]. SDC was originally developed by Professor Hong Wang in 1996, when he considered a number of challenging paper machine modelling and control problems [1]. The process and the control were presented in a PDF form. As such, the purpose of the controller design was to obtain the PDF of the controller so that the closed-loop PDF would follow the pre-specified PDF. Since then, rapid developments have been

Z. Skaf is with Control Systems Center, School of Electrical and Electronic Engineering, Sackville Street Building,University of Manchester, Manchester, M60 1QD, UK Zakwan.Skaf@manchester.ac.uk

A. AI-Bayati is with Control Systems Center, School of Electrical and Electronic Engineering, Sackville Street Building,University of Manchester, Manchester, M60 1QD, UK Ahmad.Al-Bayatiepostgrad.manchester.ac.uk

$\mathrm{H}$. Wang is with Control Systems Center, School of Electrical and Electronic Engineering, Sackville Street Building,University of Manchester, Manchester, M60 1QD, UK Hong.Wang@manchester.ac.uk

A. Wang is with Institute of Computer Sciences, Anhui University, Hefei City, P R China apwang401@126.com made and introduced in different control applications [3]. The most exciting PDF control approaches are based on the B-spline model. Moreover, multi-layer perception (MLP) neural network models have been applied to the shape control for the output PDFs [4]. Recently, a RBFNN has been used to approximate the output PDF of the system [5]. In this work, we have used RBFs instead of B-Splines which help generalize the output PDF expression and overcome the problems with B-spline-based functional approximations.

Due to the high demand for reliability and safe operation, many FTC methods were developed in the past four decades, which have the capability of detecting the occurrence of faults and maintaining the performance of the system in the presence of faults at a prescribed level [6].In most cases, the literatures on the FTC algorithms for stochastic systems have been presented under the assumption that the random variables or the noise in the stochastic system are subject to Gaussian distribution [7]. In [8], a nonlinear adaptive observer-based fault diagnosis alorithm has been presented for the SDC systems that are based on the rational squareroot B-spline approximation model. When faults occur in the system, the controller was redesigned. This method is suffering from the complexity of modelling of stochastic distribution control. As such, there is a need to develop FTC methods that can be applied to general stochastic systems subject to arbitrary variables distribution, and reduced the complexity of SDC modelling.

\section{Fault Tolerant Control}

\section{A. Problem Formulation}

Similar to [9], consider $u_{k}(i) \in R^{r}$ is the input of a discrete-time dynamic stochastic system at the $i^{\text {th }}$ time instant within the $k^{\text {th }}$ batch, $y \in[a, b]$ is the output. At sample time $k, y$ can be described by its PDF $\gamma_{k}\left(y, u_{k}(i)\right)$. Assuming that $[a, b]$ is known and the probability density function is continuous and bounded within each iteration. The well-known RBF neural networks can be used to approximate the square root of the output PDF as

$$
\sqrt{\gamma\left(y, u_{k}(i)\right)}=\sum_{l=1}^{n} \nu_{l, k}\left(u_{k}(i)\right) r_{l, k}(y)
$$

where $\gamma\left(y, u_{k}(i)\right)$ is the output PDF measured at the $i^{t h}$ time instant within the $k^{\text {th }}$ batch. Also $\nu_{l, k}(i)$ is the $l^{\text {th }}$ weight element of the RBFNN in the $i^{t h}(i=1,2, \ldots, m)$ sample time within the $k^{\text {th }}$ batch, and $r_{l, k}(y)$ denotes the $l^{\text {th }}$ $(l=1,2, \ldots, n)$ RBF activation function within $k^{t h}$ batch. Assume $n$ and $k$ represent the number of RBFs and the 
batch length, respectively. The RBF activation functions are expressed as follows [5].

$$
r_{l, k}(y)=\exp \left(-\frac{\left(y_{j}-\mu_{l, k}\right)^{2}}{2 \sigma_{l, k}^{2}}\right)
$$

where $\mu_{l, k}, \sigma_{l, k}$ are the centres and widths of the RBF basis functions within $k^{t h}$ batch, respectively.

Different from [10], [11], the output PDF described in (1) can be re-written as the following vector form.

$$
\sqrt{\gamma\left(y, u_{k}(i)\right)}=\left[\begin{array}{ll}
R_{k}(y) & r_{n, k}(y)
\end{array}\right]\left[\begin{array}{c}
V_{k}(i) \\
\nu_{n, k}(i)
\end{array}\right]
$$

where

$$
\begin{aligned}
& R_{k}(y)=\left[r_{1, k}(y), r_{2, k}(y), \ldots, r_{n-1, k}(y)\right] \\
& V_{k}(i)=\left[\nu_{l, k}(i), \nu_{2, k}(i), \ldots, \nu_{n-1, k}(i)\right]^{T} .
\end{aligned}
$$

Since, $\gamma\left(y, u_{k}(i)\right)$ is a probability density function, it must satisfy the following integral constraint [1].

$$
\int_{a}^{b}\left(\gamma\left(y, u_{k}(i)\right)\right) d y=\int_{a}^{b}\left(\sqrt{\gamma\left(y, u_{k}(i)\right)}\right)^{2} d y=1
$$

By substituting $\gamma\left(y, u_{k}(i)\right)$ with (3) and solving the equation for $\nu_{n, k}(i)$ similar to [1], [12], it can be shown that the following state constraint shall be satisfied within each batch to guarantee that the measured $\gamma\left(y, u_{k}(i)\right)$ is a probability density function [1].

$$
V_{k}^{T}(i) Q_{a b, k} V_{k}(i) \leq 1
$$

where

$$
\begin{gathered}
Q_{a b, k}=b_{1, k}-b_{3, k}^{-1} b_{2, k}^{T} b_{2, k} \\
b_{1, k}=\int_{a}^{b} R_{k}^{T}(y) R_{k}(y) d y \\
b_{2, k}=\int_{a}^{b} r_{n, k}(y) R_{k}(y) d y \\
b_{3, k}=\int_{a}^{b} r_{n, k}^{2}(y) d y
\end{gathered}
$$

It has been proven in [1] that $Q_{a b, k}$ is always positive definite. When (5) holds, it can be seen that $\nu_{n, k}$ can be represented as a known nonlinear function of $V_{k}$ called $h(V(k))$. Thus the output PDF described in (1) can be rewritten as follows.

$$
\sqrt{\gamma\left(y, u_{k}(i)\right)}=R_{k}(y) V_{k}(i)+r_{n, k}(y) h(V(k))
$$

In (6), the nonlinear function $h(V(k))$ should satisfy the following Lipschitz condition

$$
\left\|h\left(V_{1, k}(i)\right)-h\left(V_{2, k}(i)\right)\right\| \leq\left\|\bar{U}_{1}\left(V_{1, k}(i)-V_{2, k}(i)\right)\right\|
$$

where $\bar{U}_{1}$ is a known matrix.

Thus the dynamic model between the output PDF and the RBF neural network weight vectors in the presence of the actuator fault will be established as follows.

$$
\begin{aligned}
V_{k}(i+1) & =A_{k} V_{k}(i)+B_{k} u_{k}(i)+G g\left(V_{k}(i)\right)+D F_{k}(i) \\
\sqrt{\gamma\left(y, u_{k}(i)\right)} & =R_{k}(y) V_{k}(i)+r_{n, k}(y) h\left(V_{k}(i)\right)
\end{aligned}
$$

Similar to [10], the nonlinear dynamics of the model in (8) is supposed to satisfy the following Lipschitz condition.

$$
\left\|g\left(V_{1, k}(i)\right)-g\left(V_{2, k}(i)\right)\right\| \leq\left\|\bar{U}_{2}\left(V_{1, k}(i)-V_{2, k}(i)\right)\right\|
$$

where $\bar{U}_{2}$ is a known matrix.

\section{B. Fault Detection}

In order to detect the fault based on the changes of PDFs, the following nonlinear observer is considered:

$$
\begin{aligned}
& \hat{x}(k+1)=A \hat{x}(k)+B u(k)+G g(\hat{x}(k))+L \epsilon(k) \\
& \epsilon(t)=\int_{a}^{b} \mu(y)(\sqrt{\gamma(y, u(k, F))}-\sqrt{\hat{\gamma}(y, u(k))}) d y \\
& \sqrt{\hat{\gamma}(y, u(k))}=R(y) E \hat{x}(k)+h(E \hat{x}(k)) r_{n}(y)
\end{aligned}
$$

where $\hat{x}(k) \in R^{n}$ is the estimated state, $L \in R^{n \times p}$ is the filter gain to be determined. Residual $\epsilon(k)$ is formulated as an integral of the difference between the measured PDFs and the estimated ones, where, $\mu(y) \in R^{p \times 1}$ is a pre-specified weighting vector.

\section{Fault Diagnosis}

Once the fault is detected, the fault value must be estimated. For this purpose, the following observer is considered:

$$
\begin{gathered}
\hat{x}(k+1)=A \hat{x}(k)+B u(k)+G g(\hat{x}(k))+L \epsilon(k)+D \hat{F}(k) \\
\sqrt{\hat{\gamma}(y, u(k))}=R(y) E \hat{x}(k)+r_{n}(y) h(E \hat{x}(k)) \\
\hat{F}(k+1)=-\Upsilon_{1} \hat{F}(k)+\Upsilon_{2} \epsilon(k)
\end{gathered}
$$

where $\hat{F}(k)$ is the estimation of $F(k) . \Upsilon_{1}>0$ and $\Upsilon_{2}$ are the learning operators to be determined together with $L$ by the diagnosis algorithm. Apart from fault detection and diagnosis strategy, the controller design will be as follows.

\section{Controller Design}

A generalized PI controller with tuneable coefficients is considered as adaptive controller in this work as follows

$$
\begin{aligned}
& \xi_{k}(i)=\xi_{k}(i-1)+T_{s} e_{k}(i-1) \\
& u_{k}(i)=K_{P, k} \cdot e_{k}(i)+K_{I, k} \xi_{k}(i)
\end{aligned}
$$

where $e_{k}(i)=V_{g}-V_{k}(i)$ represents the dynamical weight tracking error, and $T_{s}$ is the sampling time. Substituting (12) in (8) yields the following closed-loop system for the weight control loop with the $k^{t h}$ batch:

$$
M_{k}(i+1)=\bar{A}_{k} M_{k}(i)+\bar{B}_{k} V_{g}+\bar{G} g\left(M_{k}(i)\right)+\bar{D} F_{k}(i)
$$

where

$$
\begin{aligned}
M_{k}(i) & =\left[\begin{array}{l}
V_{k}(i) \\
\xi_{k}(i)
\end{array}\right], \bar{A}_{k}=\left[\begin{array}{cc}
A_{k}-B_{k} K_{P, k} & B_{k} K_{I, k} \\
-T_{s} I & I
\end{array}\right] \\
\bar{B}_{k} & =\left[\begin{array}{c}
B_{k} K_{P, k} \\
T_{s} I
\end{array}\right], \bar{G}=\left[\begin{array}{ll}
G & 0 \\
0 & 0
\end{array}\right], \bar{D}=\left[\begin{array}{l}
D \\
0
\end{array}\right]
\end{aligned}
$$




$$
g\left(M_{k}(i)\right)=\left[\begin{array}{c}
g\left(V_{k}(i)\right) \\
0
\end{array}\right]
$$

Denote

$$
\tilde{A}_{k}=\left[\begin{array}{cc}
A_{k} & 0 \\
-T_{s} I & I
\end{array}\right], \tilde{B}_{k}=\left[\begin{array}{c}
B_{k} \\
0
\end{array}\right]
$$

The following theorem represents the solvability conditions of the general PI controller

Theorem 1. Within the $k^{\text {th }}$ batch, for the parameter $\lambda$, if there exit matrices $P>0$, and $R$ satisfying the following LMIs for any initial condition $M(0)$ satisfying constraint (5)

$\tilde{\Psi}_{k}=\left[\begin{array}{ccccccc}-P_{k} & 0 & 0 & 0 & 0 & N_{1}^{T} & N_{2}^{T} \\ 0 & -\lambda^{2} I & 0 & 0 & 0 & \bar{G}^{T} & 0 \\ 0 & 0 & M_{6} & \Upsilon_{1}^{T} & N_{3}^{T} & \bar{D}^{T} & 0 \\ 0 & 0 & \Upsilon_{1} & I & -\Upsilon_{2}^{T} & 0 & 0 \\ 0 & 0 & N_{3} & -\Upsilon_{2} & N_{4} & 0 & 0 \\ N_{1} & \bar{G} & \bar{D} & 0 & 0 & -P_{k} & 0 \\ N_{2} & 0 & 0 & 0 & 0 & 0 & -I\end{array}\right]_{(14)}$

and

$$
2 \tilde{\alpha}\left\|Q_{a b}\right\|\left\|\bar{B}_{k} V_{g}\right\|^{2} \leq \lambda_{\min }\left(P_{k}\right)
$$

where

$$
\begin{aligned}
& N_{1}=\tilde{A}_{k} P_{k}+\tilde{B}_{k} R, N_{2}=\lambda \bar{U}_{2} P_{k} \\
& N_{3}=-\Upsilon_{2}^{T} \Upsilon_{1}, N_{4}=\Upsilon_{2}^{T} \Upsilon_{2}
\end{aligned}
$$

then, the closed loop system is stable with $\lim _{k \rightarrow \infty} e(i)=0$, and the controller parameters can be calculated by using

$$
\left[\begin{array}{ll}
K_{P} & K_{I}
\end{array}\right]=R P^{-1}
$$

Proof : For this purpose, the following Lyapunov function is considered.

$$
\begin{aligned}
\Phi_{3}(i) & =M_{k}^{T}(i) P^{-1} M_{k}(i)+\bar{F}_{k}^{T}(i) \bar{F}_{k}(i) \\
& +\lambda^{2} \sum_{j=1}^{i-1}\left[\left\|\bar{U}_{2} M_{k}(i)\right\|^{2}-\left\|g\left(M_{k}(i)\right)\right\|^{2}\right]
\end{aligned}
$$

where $\bar{F}(k)=F(k)-\hat{F}(k)$

It can be verfied that

$$
\begin{aligned}
\Delta \Phi_{3} & =\Phi_{3}\left(M_{k}(i+1), i+1\right)-\Phi_{3}\left(M_{k}(i), i\right) \\
& =\tilde{M}_{k}^{T}(i) \tilde{\Psi}_{1, k} \tilde{M}_{k}(i)+2 \tilde{M}_{k}(i) \tilde{N}_{k}^{T} P_{k}^{-1} \bar{B}_{k} V_{g} \\
& +V_{g}^{T} \bar{B}^{T} P^{-1} \bar{B} V_{g}
\end{aligned}
$$

where

$$
\begin{aligned}
\tilde{\Psi}_{1, k}=\left[\begin{array}{ccccc}
\tilde{N}_{k 1} & Q_{1}^{T} & Q_{2}^{T} & 0 & 0 \\
Q_{1} & Q_{3} & Q_{4}^{T} & 0 & 0 \\
Q_{2} & Q_{4} & \tilde{N}_{k, 2} & \Upsilon_{1}^{T} & -\Upsilon_{1}^{T} \Upsilon_{2} \\
0 & 0 & \Upsilon_{1} & I & -\Upsilon_{2}^{T} \\
0 & 0 & -\Upsilon_{2}^{T} \Upsilon_{1} & -\Upsilon_{2} & \Upsilon_{2}^{T} \Upsilon_{2}
\end{array}\right] \\
\tilde{N}_{k}=\left[\bar{A}_{k}^{T}, \bar{G}_{k}^{T}, \bar{D}_{k}^{T}, 0,0\right] \\
\tilde{N}_{k, 1}=\bar{A}_{k}^{T} P_{k}^{-1} \bar{A}_{k}-P_{k}^{-1}+\lambda^{2} \bar{U}_{2}^{T} \bar{U}_{2} \\
\tilde{N}_{k, 2}=\bar{D}_{k}^{T} P_{k}^{-1} \bar{D}_{k}-I+\Upsilon_{1}^{T} \Upsilon_{1} \\
Q_{1}=\bar{G}^{T} P_{k}^{-T} \bar{A}_{k} \\
Q_{2}=\bar{D}_{k}^{T} P_{k}^{-T} \bar{A}_{k} \\
Q_{3}=\bar{G}^{T} P_{k}^{-1} \bar{G}-\lambda^{2} I \\
Q_{4}=\bar{D}_{k}^{T} P_{k}^{-T} \bar{G}
\end{aligned}
$$

and

$$
\tilde{M}_{k}^{T}(i)=\left[M_{k}^{T}(i), g^{T}\left(M_{k}(i)\right), \bar{F}_{k}^{T}, \Delta F^{T}(k), \epsilon(k)\right]
$$

By using the well-know Schur complement formula, (17) can be as follows.

$$
\tilde{\Psi}_{2, k}=\left[\begin{array}{cccccc}
Q_{5} & 0 & 0 & 0 & 0 & \bar{A}_{k}^{T} \\
0 & -\lambda^{2} I & 0 & 0 & 0 & \bar{G}^{T} \\
0 & 0 & M_{6} & \Upsilon_{1}^{T} & N_{3}^{T} & \bar{D}^{T} \\
0 & 0 & \Upsilon_{1} & I & -\Upsilon_{2}^{T} & 0 \\
0 & 0 & N_{3} & -\Upsilon_{2} & N_{4} & 0 \\
\bar{A}_{k} & \bar{G} & \bar{D} & 0 & 0 & -P_{k}
\end{array}\right]<0
$$

where $Q_{5}=-P_{k}^{-1}+\lambda^{2} \bar{U}_{2}^{T} \bar{U}_{2}$.

By pre-multiplying $\tilde{\Psi}_{2, k}$ by $\operatorname{diag}\left(P_{k}^{T}, I, I, I, I, I\right)$ and post multiplying it by $\operatorname{diag}\left(P_{k}, I, I, I, I, I\right)$, and applying the well-know Schur complement formula, the condition for stability will be as follows.

$\tilde{\Psi}_{3, k}\left[\begin{array}{ccccccc}-P_{k} & 0 & 0 & 0 & 0 & Q_{6}^{T} & Q_{7}^{T} \\ 0 & -\lambda^{2} I & 0 & 0 & 0 & \bar{G}^{T} & 0 \\ 0 & 0 & M_{6} & \Upsilon_{1}^{T} & N_{3}^{T} & \bar{D}^{T} & 0 \\ 0 & 0 & \Upsilon_{1} & I & -\Upsilon_{2}^{T} & 0 & 0 \\ 0 & 0 & N_{3} & -\Upsilon_{2} & N_{4} & 0 & 0 \\ Q_{6} & \bar{G} & \bar{D} & 0 & 0 & -P_{k} & 0 \\ Q_{7} & 0 & 0 & 0 & 0 & 0 & -I\end{array}\right]<0$

where $Q_{6}=\bar{A}_{k} P_{k}, Q_{7}=\lambda \bar{U}_{2} P_{k}$

By substituting matrices $\tilde{A}$ and $\tilde{B}$ into $\tilde{\Psi}_{3}, \tilde{\Psi}$ can be obtained.

If (14) holds, a positive scalar $\tilde{\alpha}$ exists so that $\tilde{\Psi} \leq-\tilde{\alpha} I$. Along with (13) it can be verified that

$$
\begin{aligned}
\Delta \Phi_{3} & \leq-\tilde{\alpha}\left\|\tilde{M}_{k}\right\|^{2}+2\left\|\tilde{M}_{k}\right\|\left\|\tilde{N}_{k}^{T} P^{-1} \bar{B} V_{g}\right\| \\
& +\left\|V_{g}\right\|^{2}\left\|\bar{B}^{T} P^{-1} \bar{B}\right\|
\end{aligned}
$$

It is obvious that the right- hand side of inequality is a second order degree polynomial with respect to $\left\|\tilde{M}_{k}\right\|$. Denote

$$
\tilde{\sigma}=V_{g}^{T} \bar{B}^{T} P^{-1} \bar{B} V_{g}
$$

Thus, it can be shown that $\Delta \Phi_{3} \leq 0$ holds if

$$
\mid \tilde{M}_{k} \| \geq \tilde{\alpha}^{-1}\left(\left\|\tilde{N}_{k}^{T} P^{-1} \bar{B} V_{g}\right\|+\sqrt{\left\|\tilde{N}_{k}^{T} P^{-1} \bar{B} V_{g}\right\|^{2}+\tilde{\alpha} \tilde{\sigma}}\right)
$$


which implies

$$
\begin{aligned}
&\left\|\tilde{M}_{k}\right\| \leq \max \left\{\left\|\tilde{M_{k}}(1)\right\|,\right. \\
&\left.\tilde{\alpha}^{-1}\left(\left\|\tilde{N_{k}^{T}} P^{-1} \bar{B} V_{g}\right\|+\sqrt{\left\|\tilde{N_{k}^{T}} P^{-1} \bar{B} V_{g}\right\|^{2}+\tilde{\alpha} \tilde{\sigma}}\right)\right\}
\end{aligned}
$$

This confirm that the closed-loop system is bounded and internally stable.

Based on (13) and(16) it can be shown that

$$
\begin{aligned}
\Delta \Phi_{3} & =\Phi_{3}\left(M_{k}(i+1), i+1\right)-\Phi_{3}\left(M_{k}(i), i\right) \\
& =\tilde{M}_{k}^{T}(i)\left(\bar{A}_{k}^{T} P_{k}^{-1} \bar{S}_{k}-P_{k}^{-1}\right) \tilde{M}_{k}(i) \\
& +2 \tilde{M}_{k}(i) \tilde{N}_{k}^{T} P_{k}^{-1} \bar{B}_{k} V_{g}+V_{g}^{T} \bar{B}^{T} P^{-1} \bar{B} V_{g}
\end{aligned}
$$

It can be verified that $\left(\bar{A}_{k}^{T} P_{k}^{-1} \bar{S}_{k}-P_{k}^{-1}\right)<-\tilde{\alpha}^{-1} I$ as long as $\left(\bar{A}_{k}^{T} P_{k}^{-1} \bar{S}_{k}-P_{k}^{-1}\right)<0$ is guaranteed by (14) Thus it can be seen that

$$
\begin{aligned}
\Delta \Phi_{3} & \leq-\tilde{\alpha}^{-1}\left\|\tilde{M}_{k}(i)\right\|^{2}+2 \tilde{M}_{k}(i) \tilde{N}_{k}^{T} P_{k}^{-1} \bar{B}_{k} V_{g} \\
& +V_{g}^{T} \bar{B}^{T} P^{-1} \bar{B} V_{g} \\
& \leq-\tilde{\alpha}^{-1}\left\|\tilde{M}_{k}(i)\right\|^{2}-\left(P_{k}^{-1 / 2} \tilde{N}_{k}^{T} \tilde{M}_{k}(i)\right. \\
& \left.-P_{k}^{-1 / 2} \bar{B}_{k} V_{g}\right)^{T}\left(P_{k}^{-1 / 2} \tilde{N}_{k}^{T} \tilde{M}_{k}(i)-P_{k}^{-1 / 2} \bar{B}_{k} V_{g}\right) \\
& +2 V_{g}^{T} \tilde{N}_{k}^{T} P_{k}^{-1} \bar{B}_{k} V_{g} \\
& \leq-\tilde{\alpha}^{-1}\left\|\tilde{M}_{k}(i)\right\|^{2}+2 \lambda_{\max }\left(P^{-1}\right)\left\|\bar{B}_{k} V_{g}\right\|^{2}
\end{aligned}
$$

Denote

$$
\tilde{\beta}=2 \lambda_{\max }\left(P^{-1}\right)\left\|\bar{B}_{k} V_{g}\right\|^{2}
$$

Then it can be shown that $\Delta \Phi_{3}<0$ holds if

$$
\left\|\tilde{M}_{k}(i)\right\|^{2}>\tilde{\alpha} \tilde{\beta}
$$

From the constraint (5) it can be seen that

$$
\begin{aligned}
V_{k}^{T}(i) Q_{a b} V_{k}(i) & \left.<\| V_{k}(i)\right)\left\|^{2}\right\| Q_{a b} \| \\
& \leq\left\|\tilde{M}_{k}(i)\right\|^{2}\left\|Q_{a b}\right\| \\
& \leq \tilde{\alpha} \tilde{\beta}\left\|Q_{a b}\right\| \leq 1
\end{aligned}
$$

Thus, the constraint in (15) can be guaranteed by the obtained results from (25) and (26) To discuss the system tracking performance, suppose that $\tilde{\varphi}_{1}(i)$ and $\tilde{\varphi}_{2}(i)$ are two trajectories of the nonlinear closed-loop system (13) corresponding to fixed initial conditions and fault, and $V_{g}$ is the input. Denote the error between the two trajectories $\chi(i)=\tilde{\varphi}_{1}(i)-\tilde{\varphi}_{2}(i)$ with $\chi(1)=0$. Then, the dynamic of $\chi(i+1)$ can be presented as follows.

$$
\chi(i+1)=\bar{A}_{k} \chi(i)+\bar{G}\left[g\left(\tilde{\varphi}_{1}\right)-g\left(\tilde{\varphi}_{2}\right)\right]
$$

The following Lyapunov function will be considered

$$
\begin{gathered}
\Phi_{4}\left(\chi(i), \tilde{\varphi}_{1}(i), \tilde{\varphi}_{2}(i), i\right)=\chi^{T}(i) P^{-1} \chi(i) \\
+\lambda^{2} \sum_{j=1}^{i-1}\left[\|U \chi(i)\|^{2}-\left\|g\left(\tilde{\varphi}_{1}(i)\right)-g\left(\tilde{\varphi}_{2}(i)\right)\right\|^{2}\right]
\end{gathered}
$$

It can be verIfied that

$$
\Delta \Phi_{4}=\tilde{\chi}^{T}(i) \tilde{\Psi}_{4, k} \tilde{\chi}(i)<-\tilde{\alpha} \mid \tilde{\chi}(i) \|
$$

where

$$
\begin{gathered}
\tilde{\chi}(i)=\left[\chi^{T}(i), g\left(\tilde{\varphi}_{1}\right)-g\left(\tilde{\varphi}_{2}\right)\right] \\
\tilde{\Psi}_{4, k}=\left[\begin{array}{cc}
\bar{A}_{k}^{T} P_{k}^{-1} \bar{A}_{k}-P_{k}^{-1}+\lambda^{2} \bar{U}_{2}^{T} \bar{U}_{2} & \bar{A}_{k}^{T} P_{k}^{-1} \bar{G}_{k} \\
\bar{G}_{k}^{T} P_{k}^{-T} \bar{A}_{k} & \bar{G}_{k}^{T} P_{k}^{-1} \bar{G}_{k}-\lambda^{2} I
\end{array}\right]
\end{gathered}
$$

This means that the closed loop system is exponentially stable around $\chi=0$ neighborhood. Thus, the tracking performance of the system has been satisfied.

\section{TUNing OF RADiAL BASIS FUnCTION}

Similar to [9], the following P-type ILC law will be used to tune the basis function parameters (RBF centres and widths) between any two batches

$$
\begin{aligned}
\mu_{l, k} & =\mu_{l, k-1}+\Lambda_{\mu} E_{k-1} \\
\sigma_{l, k} & =\sigma_{l, k-1}+\Lambda_{\sigma} E_{k-1}
\end{aligned}
$$

where the performance indices of the $(k-1)^{t h}$ batch will be as follows.

$$
E_{k-1}=\left[J_{k-1}(1), J_{k-1}(2), \ldots, J_{k-1}(m)\right]^{T}
$$

where $m$ represents the total number of time instants within a batch. $J_{k-1}(i)$ is the performance at the $i^{t h}$ sampling instant of the $(k-1)^{t h}$ batch, it can be expressed as follows.

$$
J_{k-1}(i)=\int_{a}^{b}\left(\sqrt{\gamma\left(y, u_{k}(i)\right)-\sqrt{g(y)}}\right)^{2} d y
$$

In addition, the learning parameters in (30) are defined as

$$
\begin{aligned}
& \Lambda_{\mu}=\alpha_{\mu}\left[\lambda_{1}, \lambda_{2}, \ldots, \lambda_{m}\right] \\
& \Lambda_{\sigma}=\alpha_{\sigma}\left[\bar{\lambda}_{1}, \bar{\lambda}_{2}, \ldots, \bar{\lambda}_{m}\right]
\end{aligned}
$$

where $\lambda, \bar{\lambda}$ are the learning elements, and $\alpha_{\mu}, \alpha_{\sigma}$ are the learning rates to be determined.

\section{Convergence Analysis}

Similar to [9], the learning vectors in (31) should be selected carefully to ensure the convergence of the ILC-based tuning algorithm between batches. Therefore, the closed loop performance should satisfy the following condition between batches

$$
\frac{F_{k}}{F_{k-1}}=\frac{\sum_{i=1}^{m} J_{k}(i)}{\sum_{i=1}^{m} J_{k-1}(i)} \leq 1
$$

where

$$
F_{k}=\sum_{i=1}^{m} J_{k}(i)
$$

where $F_{k}$ is the measure of the overall closed loop performance within the $k^{t h}$ batch. Since $J_{k}(i)$ is non-negative, it can be verified that

$$
\Delta F_{k}=F_{k}-F_{k-1} \leq 0
$$

The conditions of convergence have been discussed in [5], which can be summarized as follows:

$$
\sum_{i=1}^{m} \int_{a}^{b}\left[\left(\sqrt{\gamma_{k-1, i}(y)}-\sqrt{g(y)}\right) \Delta \sqrt{\gamma_{k-1, i}(y)}\right] d y \leq 0
$$




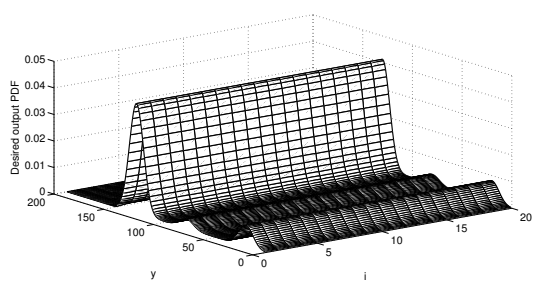

Fig. 1. Desired output PDF

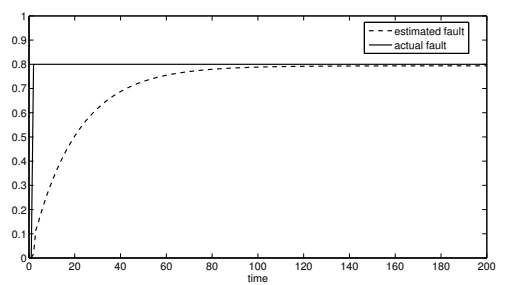

Fig. 2. Fault and its estimation under the filter

together with

$$
\begin{aligned}
\Delta R_{l, k-1}(y) & =\frac{y_{j}-\mu_{k-1}}{\sigma_{k-1}^{2}} R_{l, k-1}(y) \Lambda_{\mu} E_{k-1} \\
& +\frac{\left(y_{j}-\mu_{k-1}\right)^{2}}{\sigma_{k-1}^{3}} R_{l, k-1}(y) \Lambda_{\mu} E_{k-1}
\end{aligned}
$$

and

$$
\begin{aligned}
\Delta \sqrt{\gamma_{k-1, i}(y)} & =\sqrt{\gamma_{k-1, i}(y)}-\sqrt{\gamma_{k-2, i}(y)} \\
& =\sum_{l=1}^{n} V_{l}(i) \Delta R_{l, k-1}(y)
\end{aligned}
$$

where

$$
\begin{aligned}
\Delta R_{l, k-1}(y) & =R_{l, k-1}(y)-R_{l, k-2}(y) \\
\Delta \mu_{k} & =\mu_{k}-\mu_{k-1} \\
\Delta \sigma_{k} & =\sigma_{k}-\sigma_{k-1}
\end{aligned}
$$

\section{An Illustrated Example}

For a stochastic system with non-Gaussian process, it is supposed that the output PDF can be formulated by using three-layer neural network with three radial basis activation functions with the following initial conditions over its definition interval $[0,2]$.

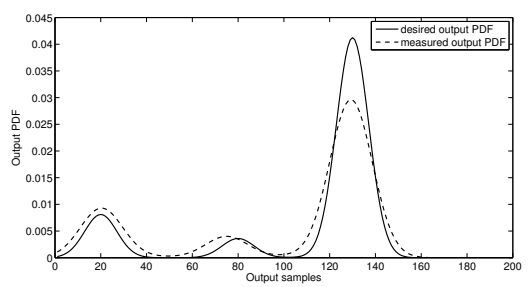

Fig. 3. 2D plot of measured and desired PDF, $k=2$

$$
\begin{gathered}
\mu_{1}=0.5, \mu_{2}=1.0, \mu_{3}=1.5 \\
\sigma_{1}=\sigma_{2}=\sigma_{3}=0.2
\end{gathered}
$$

The weight vector behaves dynamically with the following parameters:

$$
\begin{gathered}
A=\left[\begin{array}{cc}
-0.45 & 0.03 \\
0.1 & -0.28
\end{array}\right], B=\left[\begin{array}{cc}
0.45 & 0.01 \\
0.01 & -0.86
\end{array}\right], \\
G=\left[\begin{array}{cc}
0.02 & 0 \\
0 & 0.01
\end{array}\right], D=E=\left[\begin{array}{ll}
1 & 0 \\
0 & 1
\end{array}\right]
\end{gathered}
$$

The nonlinear function was chosen as follows.

$$
g(V(t))=\left[\begin{array}{c}
0 \\
\sqrt{v_{1}^{2}+v_{2}^{2}}
\end{array}\right]
$$

The initial value of the weight vector is set as $V_{1}(0)=$ $[0.001,0.001]^{T}$, and the ILC learning rates are defined as

$$
\Lambda_{\mu}=\left[\begin{array}{ccc}
-7.8 & 0 & 0 \\
0 & -13.1 & 0 \\
0 & 0 & -16.9
\end{array}\right]\left[\begin{array}{l}
0.0030 \times j \\
0.0015 \times j \\
0.0015 \times j
\end{array}\right]
$$

along with

$$
\Lambda_{\sigma}=\left[\begin{array}{ccc}
-0.71 & 0 & 0 \\
0 & -0.71 & 0 \\
0 & 0 & -0.71
\end{array}\right]\left[\begin{array}{l}
0.005 \times j \\
0.005 \times j \\
0.005 \times j
\end{array}\right]
$$

where $j=0,1, \ldots, 20$. In addition, the matrices $U_{1}$ and $U_{2}$ were chosen as follows.

$$
U_{1}=\left[\begin{array}{cc}
0.1 & 0 \\
0 & 0.1
\end{array}\right], U_{2}=\left[\begin{array}{ll}
1 & 0 \\
0 & 1
\end{array}\right]
$$

For simulation purposes, 200 uniformly distributed samples of the output and 20 for the time samples were used. Also, the RBF basis functions parameters of the desired output PDF are as follows:

$$
\begin{gathered}
\mu_{g 1}=0.2, \mu_{g 2}=0.8, \mu_{g 3}=1.3 \\
\sigma_{g 1}=\sigma_{g 2}=\sigma_{g 3}=0.1
\end{gathered}
$$

Moreover, the desired dynamical weights are set as $V_{g}=$ $[0.09 ; 0.06]$. With the above parameters, the 3-D plot of the desired PDF shape is shown in Fig. 1. Assume that the modelling error satisfies $|\omega(y, u(k), F)| \leq 0.002$. The bound of medelling error satisfies $\tilde{\delta}=0.0008$ for $\mu(y)=1$. It can be compute that

$$
\begin{aligned}
& \Lambda_{1}=\left[\begin{array}{ll}
0.3010 & 0.0347 \\
0.0347 & 0.3010
\end{array}\right], \Lambda_{2}=\left[\begin{array}{ll}
0.0001 & 0.0350
\end{array}\right], \\
& \Lambda_{3}=0.3413
\end{aligned}
$$

Also, it can be seen that

$$
\Gamma_{1}=\left[\begin{array}{ll}
0.0389 & 0.0348
\end{array}\right], \Gamma_{2}=0.4225
$$

To demonstrate the effectiveness of the proposed algorithm, the fault is chosen to be a constant signal as $F(t)=0.8$, and it is supposed to commence at $T=2 \mathrm{~s}$.

By applying the nonlinear fault isolation filter, the estimated fault should track the real fault profile as close as possible. Fig. 2 shows that such a filter can effectively diagnose the actuator fault. 


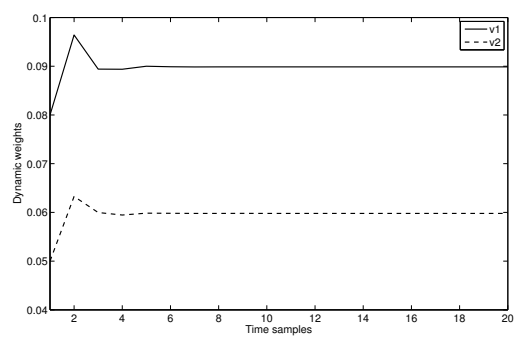

Fig. 4. Control weight under FTC

The difference of the PDF tracking within the second batch between the measured and desired PDF is shown in Fig. 3. The parameters of controller in this batch are

$$
K_{P}=\left[\begin{array}{cc}
-5522.4 & -3534.0 \\
7431.7 & 4453.3
\end{array}\right], K_{I}=\left[\begin{array}{ccc}
0.00394 & 0.00394 \\
0.04860 & 0.04860
\end{array}\right]
$$

After the final batch $(k=10)$, the coefficients of PI controller are as follows.

$K_{P}=\left[\begin{array}{cc}175.88 & -275.59 \\ -307.94 & 482.51\end{array}\right], K_{I}=\left[\begin{array}{cc}0.1876 & 0.1876 \\ -0.3285 & -0.3285\end{array}\right]$

As a result, the corresponding of the weight control loop with the last batch of operation can be described in Fig. 4 . Fig. 4 clearly demonstrates the effect of the fault-tolerant action on maintaining the correct value for the controlled weights. Moreover, it reflects the effectiveness of LMI feasbility results.

The 3D mesh plot of the output PDF in the last batch of operation is shown in Fig. 5. In addition, the PDF tracking performance within the last batch of operation is shown in Fig. 6.

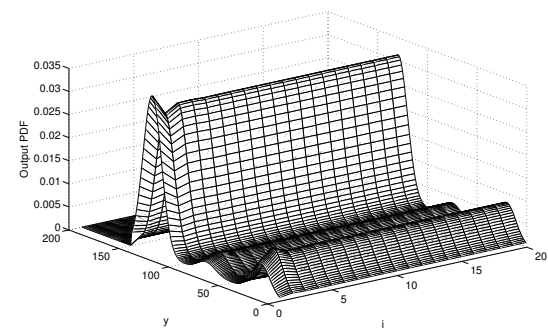

Fig. 5. 3-D mesh plot of the measured output PDF after the fault happens

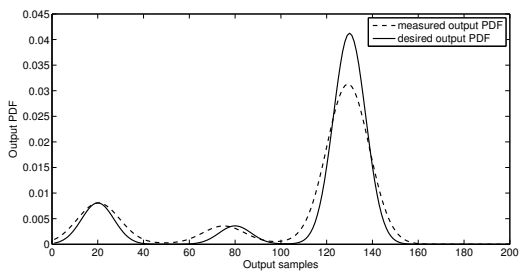

Fig. 6. 2D plot of measured and desired PDF, $\mathrm{k}=10$
Finally, the trend of the ILC performance function along the batches implies the effectiveness of the proposed algorithm is shown in Fig. 7

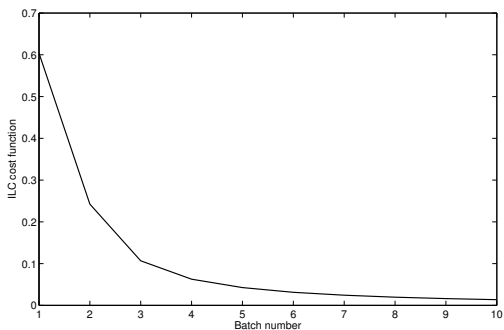

Fig. 7. performance function of the ILC

\section{ACKNOWLEDGMENTS}

This work is jointly supported by the Chinese National Fundamental Research Program (No. 2009CB320604), the National Natural Science of China (No. 61074071,60534010), UK EPSRC (EP/I016643/1, EP/E050441/1-CICADA), the Funds for Creative Research Groups of China (No.60821063), and 111 Project (No. B08015).

\section{REFERENCES}

[1] H. Wang, Bounded dynamic Stochastic Systems: Modelling and Control. London: Springer-Verlag, 2000.

[2] A. Wang, P. Afshar, and H. Wang, "Complex stochastic systems modelling and control via iterative machine learning," Neurocomputing, vol. 71, no. 13-15, pp. 2685-2692, 2008.

[3] A. Wang, H. Wang, and L. Guo, "Recent advances on stochastic distribution control: Probability density function control," in Control and Decision Conference, 2009. CCDC '09. Chinese, pp. xxxv-xli, 2009

[4] H. Wang, "Multivariable output probability density function control for non-gaussian stochastic systems using simple mlp neural networks," Proceedings of the IFAC International Conference on Intelligent Control Systems and Signal Processing, Algarve, Portugal, pp. 8489, 2003.

[5] H. Wang and P. Afshar, "Ilc-based fixed-structure controller design for output pdf shaping in stochastic systems using $1 \mathrm{mi}$ techniques," IEEE transactions on automatic control, vol. 54, no. 4, pp. 760-773, 2009.

[6] R. Iserman, Fault-Diagnosis systems: An Introduction from Fault Detection to Fault Tolerance. Berlin Heidelberg: Springer-Verlag, 2006.

[7] R. Srichander and B. K. Walker, "Stochastic stability analysis for continuous time fault tolerant control systems," in International Journal of Control, vol. 57, pp. 433-452, 1993.

[8] L.-N. Yao, A. Wang, and H. Wang, "Fault detection, diagnosis and tolerant control for non-gaussian stochastic distribution systems using a rational square-root approximation model," International Journal of Modelling, Identification and Control, vol. 3, no. 2, pp. 162-172, 2008.

[9] H. Wang, P. Afshar, and H. Yue, "Ilc-based generalised pi control for output pdf of stochastic systems using lmi and rbf neural networks," Proceedings of the IEEE Conference on Decision and Control, pp. 5048-5053, 2006.

[10] L. Guo and H. Wang, "Fault detection and diagnosis for general stochastic systems using b-spline expansions and nonlinear filters," IEEE Transactions on Circuits and System, vol. 52, no. 8, pp. 1644$1652,2005$.

[11] H. Wang and W. Lin, "Applying observer based fdi techniques to detect faults in dynamic and bounded stochastic distributions," International Journal of Control, vol. 73, pp. 1424-1436, 2000.

[12] L. Guo and H. Wang, "Applying constrained nonlinear generalized pi strategy to pdf tracking control through square root b-spline models," International Journal of Control, vol. 77, no. 17, pp. 1481-1492, 2004. 